\title{
Evaluation of psoralen ethosomes for topical delivery in rats by using in vivo microdialysis
}

This article was published in the following Dove Press journal:

International Journal of Nanomedicine

23 January 2014

Number of times this article has been viewed

\section{Yong-Tai Zhang \\ Li-Na Shen \\ Ji-Hui Zhao \\ Nian-Ping Feng}

Department of Pharmaceutical Sciences, Shanghai University of

Traditional Chinese Medicine,

Shanghai, People's Republic of China
Correspondence: Nian-Ping Feng Department of Pharmaceutics, School of Pharmacy, Shanghai University of

Traditional Chinese Medicine, 1200 Cailun Road, Zhangjiang Hi-Tech Park, Pudong New District, Shanghai 20I203, People's Republic of China

Tel +862151322198

Fax + 86 21 5I32 2198

Email npfeng@hotmail.com
Abstract: This study aimed to improve skin permeation and deposition of psoralen by using ethosomes and to investigate real-time drug release in the deep skin in rats. We used a uniform design method to evaluate the effects of different ethosome formulations on entrapment efficiency and drug skin deposition. Using in vitro and in vivo methods, we investigated skin penetration and release from psoralen-loaded ethosomes in comparison with an ethanol tincture. In in vitro studies, the use of ethosomes was associated with a 6.56-fold greater skin deposition of psoralen than that achieved with the use of the tincture. In vivo skin microdialysis showed that the peak concentration and area under the curve of psoralen from ethosomes were approximately 3.37 and 2.34 times higher, respectively, than those of psoralen from the tincture. Moreover, it revealed that the percutaneous permeability of ethosomes was greater when applied to the abdomen than when applied to the chest or scapulas. Enhanced permeation and skin deposition of psoralen delivered by ethosomes may help reduce toxicity and improve the efficacy of long-term psoralen treatment.

Keywords: absorption enhancer, formulation vehicle, nanocarriers, nanoparticles, transdermal

\section{Introduction}

Psoralen, a small molecule that belongs to the coumarin family of compounds, is isolated from Fructus psoraleae, a common herb used in traditional Chinese medicine. Psoralen is a photosensitive compound that is used to treat vitiligo and psoriasis through exposure to sunlight or ultraviolet radiation, and psoralen ultraviolet A (PUVA) therapy has been approved by the US Food and Drug Administration for clinical use. ${ }^{1-3}$ PUVA is an important treatment for refractory psoriasis. ${ }^{4}$ More and more dermatologists across the world have started to prescribe PUVA therapy, and for many patients, it has become the first-choice treatment for the effective control of psoriasis. ${ }^{5,6}$ Treatment with psoralen may increase tyrosinase activity and promote melanin synthesis in normal melanocytes adjacent to the damaged cells. ${ }^{7,8}$ The conventional formulations of psoralen, such as tinctures and ointments, have weak percutaneous permeability and poor deposition in the skin, which usually leads to frequent administration and may cause adverse reactions..$^{9,10}$ Topical delivery of drugs by liposomal formulations has attracted considerable interest in recent decades because of the improved therapeutic effects. However, conventional liposomes do not permeate deeply into the skin. Their particle-size distribution is uneven and they remain confined to the epidermis. ${ }^{11,12}$ Touitou et al discovered a novel type of liposomes, namely, ethosomes. ${ }^{13}$ Ethosomes are composed of phospholipids, water, and a high concentration of ethanol, usually about 
$20 \%$ to $45 \%$. ${ }^{13}$ Owing to a liquid and flexible lipid bilayer, ethosomes are easily deformable and may permeate deep into the skin, thus enhancing drug delivery. ${ }^{14,15}$ Moreover, they increase drug deposition in the skin and enhance its permeability in scar tissue. ${ }^{16-19}$

Microdialysis is a minimally invasive in vivo sampling technique for measuring the concentrations of endogenous and exogenous substances in the extracellular space of tissues and organs. ${ }^{20}$ Dialysis is the process of sampling fluid in the in vivo environment by using a semipermeable membrane that allows biological molecules to diffuse into a sample via a concentration gradient. ${ }^{21}$ The preliminary results have demonstrated the ability of microdialysis probes to continuously monitor the concentrations of drugs in the dermis in vivo. ${ }^{22}$ Microdialysis has also been successfully used to obtain reliable pharmacokinetic data for routine estimation of cutaneous permeation. ${ }^{23}$

In the present study, we employed in vivo microdialysis to compare a novel transdermal drug delivery using ethosomes with one using a conventional tincture. We aimed to establish whether ethosomes provided better permeability and skin deposition of psoralen than those provided by conventional modes of delivery, which might help achieve better efficacy of psoralen treatment in the future. Ethosomes with different chemical compositions were prepared and analyzed in vitro. They were then used as vehicles to enhance the delivery and skin deposition of psoralen after topical administration to different skin sites in rats, including the abdomen, chest, and scapular region. Microdialysis was used to monitor the psoralen concentration in vivo.

\section{Materials and methods Materials}

Lipoid S 100 (phosphatidylcholine [PC] from soybean lecithin), containing 95.8\% PC, was purchased from Lipoid $\mathrm{GmbH}$ (Ludwigshafen, Germany). Psoralen with purity exceeding 98.0\% (isolated from F. psoraleae) was supplied by Ze-lang BioScience (Nanjing, People's Republic of China). All other chemicals were of high-performance liquid chromatography (HPLC) or analytical grade and were obtained from Sinopharm Chemical Reagent Co, Ltd (Shanghai, People's Republic of China).

\section{Animals}

Male Sprague Dawley rats, weighing 180-220 g, were used. The study was approved by the Animal Ethical Committee, Shanghai University of Traditional Chinese Medicine (permit number SYXK [Hu] 2009-0069). Animals were kept in an agreeable environment with free access to rodent diet and water, and were acclimatized for at least 1 week before the study.

\section{HPLC assay}

The LC-2010A HT Liquid Chromatograph system (Shimadzu Corporation, Kyoto, Japan) was used to determine the concentration of psoralen in samples. The HPLC system consisted of a double-plunger pump (Shimadzu Corporation), an autosampler (Shimadzu Corporation), an online degasser (Shimadzu Corporation), a Diamonsil $\mathrm{C} 18$ reverse phase column $(5 \mu \mathrm{m}, 4.6 \mathrm{~mm}$ inner diameter $\times$ 25 cm; Dikma Technologies, Inc, Lake Forest, CA, USA), a column oven (Shimadzu Corporation), an ultraviolet detector (Hamamatsu Photonics, Hamamatsu, Japan), and a recording integrator (Shimadzu Corporation). The mobile phase was methanol:water (55:45, volume/volume $[\mathrm{v} / \mathrm{v}])$ with a flow rate of $1 \mathrm{~mL} / \mathrm{min}$. The column temperature was constant at $35^{\circ} \mathrm{C}$, and the detection wavelength was 246 nm. Percentage recoveries ranged from $97.4 \%$ to $102.8 \%$. The psoralen intra- and interday relative standard deviation values were $0.87 \%$ and $2.15 \%$, respectively. Samples from in vitro experiments were filtered through a disposable nylon syringe filter (pore size, $0.45 \mu \mathrm{m}$ ) with a $13-\mathrm{mm}$ diameter (Shanghai Anpel Scientific Instrument Co, Ltd, Shanghai, People's Republic of China) before automatic injection into the HPLC system. Samples from in vivo microdialysis were directly assayed in a timely manner without any handling.

\section{Preparation and comparison of ethosomes}

Ethosome formulations, labeled from ES1 to ES5, were composed of different levels of Lipoid S 100 and ethanol, as listed in Table 1. A simple modified injection method was used to prepare a colloidal ethosome suspension. In a closed environment, Lipoid S 100 and psoralen were dissolved in ethanol. ${ }^{13}$ The required amount of purified water was injected at a flow rate of $12 \mathrm{~mL} / \mathrm{h}$ with a WZ-50C6 microinfusion pump (Smiths Medical, Norwell, MA, USA) into the ethanol solution, with constant mixing at $700 \mathrm{rpm}$ (IKA Works $\mathrm{GmbH}$ and Co, Staufen, Germany). After water injection, magnetic stirring was continued for 5 minutes at $300 \mathrm{rpm}$ to ensure mixing uniformity. Ethosome suspensions were probe-sonicated for 5 minutes (power, $900 \mathrm{~Hz}$; quiescent interval, 3 seconds) in an ice-water bath by using an ultrasonic cell disruption system (Scientz Biotechnology Co, Ltd, Ningbo, People's Republic of China). A uniform design containing two factors and five levels was used for optimization of ethosome formulations, with Lipoid S 100 content (weight/volume [w/v], \%) as factor $\mathrm{X}_{1}$ 
Table I Composition and in vitro evaluation of ethosomes (ESI-ES5) tested for optimal skin permeation via the $U_{5}\left(5^{2}\right)$ uniform experimental design, with the tincture as the compared formulation (mean \pm standard deviation, $n=3$ )

\begin{tabular}{|c|c|c|c|c|c|c|}
\hline Ethosomes & $\begin{array}{l}\frac{\text { Factor A }\left(X_{1}\right)}{\text { Lipoid S } 100} \\
(w / v, \%)\end{array}$ & $\begin{array}{l}\text { Factor B }\left(X_{2}\right) \\
\text { Ethanol } \\
(v / v, \%)\end{array}$ & $\begin{array}{l}\text { Drug loaded } \\
(w / v, \%)\end{array}$ & $\begin{array}{l}\text { Particle } \\
\text { size }(\mathrm{nm})\end{array}$ & $\begin{array}{l}\text { Response }\left(Y_{1}\right) \\
\text { Entrapment } \\
\text { efficacy }(\%)\end{array}$ & $\begin{array}{l}\text { Response }\left(\mathrm{Y}_{2}\right) \\
\text { Deposition in } \\
\text { skin }\left(\mu \mathrm{g} / \mathrm{cm}^{2}\right)\end{array}$ \\
\hline ESI & 4.0 & 30 & 2.0 & $\mid 46.27 \pm 18.88$ & $59.19 \pm 8.16$ & $0.35 \pm 0.04$ \\
\hline ES2 & 5.0 & 40 & 2.0 & $120.77 \pm 22.43$ & $85.62 \pm 0.76$ & $3.6 I \pm 1.21 *$ \\
\hline ES3 & 6.0 & 25 & 2.0 & $|47.37 \pm| 7.04$ & $92.03 \pm 8.95$ & $2.84 \pm 1.46^{*}$ \\
\hline ES4 & 7.5 & 35 & 2.0 & $\mid 59.07 \pm 25.97$ & $38.56 \pm 5.94$ & $0.72 \pm 0.18^{*}$ \\
\hline ES5 & 8.0 & 45 & 2.0 & $56.71 \pm 1.02$ & $18.30 \pm 2.29$ & $1.98 \pm 0.90 *$ \\
\hline Tincture & - & 70 & 2.0 & - & - & $0.55 \pm 0.18$ \\
\hline
\end{tabular}

Note: Compared with the tincture, $* P<0.05$.

Abbreviation: ES, ethosomes.

and ethanol content (v/v, \%) as factor $\mathrm{X}_{2}$. The experimental responses were defined as $Y_{1}$ for entrapment efficiency (EE) and $\mathrm{Y}_{2}$ for drug skin deposition (DSD).

The tincture was prepared by adding psoralen at a concentration of $0.2 \%(\mathrm{w} / \mathrm{v})$ to $70 \%$ ethanol (in purified water, $\mathrm{v} / \mathrm{v})$.

\section{Characterization of ethosomes}

The average particle size of the ethosomes was measured using dynamic light scattering with a computerized Malvern Autosizer Nano ZS90 inspection system (Malvern Instruments Ltd, Malvern, UK). The measurements were performed in triplicate. The appearance of the ethosomes was examined using a transmission electron microscope (Philips Tecnai 12; Philips, Amsterdam, the Netherlands). Samples were prepared for negative staining as follows: copper nets carrying formvar-supporting film (200 mesh; Zhong Jing Ke Yi Technology Inc, Beijing, People's Republic of China) were placed on a stencil plate. Ethosomes were gently dropped on the film, and the film was allowed to dry for about 20 minutes. A drop of uranyl acetate $(2 \%)$ was added to the film and allowed to dry for 10 minutes. The film was then observed under a transmission electron microscope.

\section{Entrapment efficiency of ethosomes}

A dialysis method was used to determine the EE of ethosomes. A dialysis bag with a molecular weight cut-off of 10,000 (Qingyang Copalyton Biotechnology, Shanghai, People's Republic of China) was soaked in boiling purified water for 30 minutes, and $0.5 \mathrm{~mL}$ of the ethosome suspension was placed in the dialysis bag. The bag was then closed using dialysis tubing closures and immersed in $200 \mathrm{~mL}$ of $20 \%$ polyethylene glycol $400(\mathrm{v} / \mathrm{v})$. The receiver medium was stirred at $300 \mathrm{rpm}$ by using a magnetic stirrer for approximately 5 hours. Psoralen in the receiver medium was then assayed using HPLC, and the EE was calculated using Equation 1, where $D_{t}$ is the total amount of psoralen in the ethosome suspension and $\mathrm{D}_{\mathrm{d}}$ is the amount of the drug that diffused into the receiver medium. ${ }^{24}$

$$
\mathrm{EE}=\left(\mathrm{D}_{\mathrm{t}}-\mathrm{D}_{\mathrm{d}}\right) / \mathrm{D}_{\mathrm{t}}
$$

The results were reported as the mean of three independent experiments performed in triplicate.

\section{In vitro skin permeation studies}

In vitro permeation experiments were conducted using a Franz diffusion cell (Fulansi Electronic Science and Trade Co, Ltd, Tianjin, People's Republic of China) fitted with excised rat skin, and the permeation of ethosomes was compared to that of the alcohol tincture. ${ }^{25}$ Each donor compartment had a diffusion area of $1.77 \mathrm{~cm}^{2}$, and $2 \mathrm{~mL}$ of ethosomes or tincture was added to each compartment. Each receptor compartment had a volume of $16 \mathrm{~mL}$ and was filled with freshly prepared polyethylene glycol $400(20 \%)$ in normal saline $(2: 8, \mathrm{v} / \mathrm{v})$ to provide sink conditions. The receptor compartment solution was held at $32^{\circ} \mathrm{C} \pm 0.5^{\circ} \mathrm{C}$ by using a thermostat, and stirred at $300 \mathrm{rpm}$ by a magnetic bar. Each experiment was performed in triplicate. At predetermined time points, $1 \mathrm{~mL}$ samples were removed from each receptor compartment and the volume was replenished with an equal volume of receptor fluid equilibrated to $32^{\circ} \mathrm{C} \pm 0.5^{\circ} \mathrm{C}$. The concentration of psoralen in the obtained samples was determined using HPLC. The permeation profile of psoralen was obtained by plotting the mean cumulative permeation per $\mathrm{cm}^{2}$ of ethosomes or tincture against time. A linear regression analysis was performed to determine the transdermal flux $\left(\mathrm{Js}, \mu \mathrm{g} / \mathrm{cm}^{2} / \mathrm{h}\right)$ of psoralen.

\section{Determination of skin deposition}

At the end of the 24 hours in vitro skin permeation studies, skin samples were taken from the diffusion cell, and their surfaces were washed three times with fresh receptor fluid. 
Then, the samples were cut into small pieces and placed in manual glass homogenizers along with $1 \mathrm{~mL}$ of fresh receptor fluid. The homogenates were centrifuged (Eppendorf, Hamburg, Germany) at 5,000 rpm for 10 minutes, and the supernatants were transferred to $2 \mathrm{~mL}$ centrifuge tubes. After transfer, $1 \mathrm{~mL}$ of ethyl acetate was added to each sample and they were mixed for 3 minutes by using a vortexer (UniEquip $\mathrm{GmbH}$, Freital/Dresden, Germany). The samples were then centrifuged at 5,000 rpm for 10 minutes. The upper organic phase was removed, and $1 \mathrm{~mL}$ of fresh ethyl acetate was added into the same sample. The above process was then repeated. The resulting two supernatants were pooled and dried under nitrogen by using a pressure blowing concentrator (Shanghai ANPEL Scientific Instrument Co, Ltd). The sample residues were dissolved in $200 \mu \mathrm{L}$ of methanol and vortexed for 3 minutes. Finally, the samples were centrifuged at 12,000 rpm for 10 minutes, and the supernatants were assayed for psoralen by using HPLC.

\section{Microdialysis}

The microdialysis system consisted of a WZ-50C6 microinfusion pump (Smiths Medical) with a $20 \mathrm{~mL}$ plastic syringe and a linear microdialysis probe. Spectra/Por ${ }^{\circledR}$ microdialysis hollow fibers (Spectrum Laboratories, Inc, Houston, TX, USA) were prepared using regenerated cellulose (inner diameter, $200 \mu \mathrm{m}$; outer diameter, $280 \mu \mathrm{m}$; molecular weight cut-off, $13,000 \mathrm{Da})$. The fibers were glued to quartz capillary tubing (Welch Materials, Inc, Shanghai, People's Republic of China) with cyanoacrylate adhesive $\left(\right.$ Mxbon $^{\circledR}$ Super Glue; Cartell Chemical Co, Ltd, Chia-Yi Hsien, Taiwan) to create linear microdialysis probes. The inlet tube of the probes was connected to the microinjection pump using polyethylene tubing. In all the experiments, the length of the membrane accessible to dialysis was $20 \mathrm{~mm}$, and the perfusate flow rate was $3.33 \mu \mathrm{L} /$ minutes. Cannulas were used as insertion guides, and vials were used to collect the dialysate samples. Standard solutions of psoralen (STD) were prepared by dissolving pure psoralen in ethanol solution $(10 \%, \mathrm{v} / \mathrm{v})$.

\section{Recovery validation in vitro and correction in vivo}

In vitro recovery was estimated prior to the onset of microdialysis studies to ensure that the retrodialysis method was suitable for this in vivo recovery study and to ensure that the probes would provide reproducible and efficient sampling. A linear probe was placed in a $50 \mathrm{~mL}$ beaker containing different concentrations of psoralen in sequence (Table 2), and the dialysis membrane portion of the probe was completely immersed in the solution at room temperature. The probe was perfused with ethanol $(10 \%, \mathrm{v} / \mathrm{v})$ at a flow rate of $3.33 \mu \mathrm{L} /$ minutes. After an equilibration period of 30 minutes, the dialysate was collected into a small vial for 30 minutes. Dialysate samples were analyzed using HPLC to determine the psoralen concentration. Relative recovery (RR) was calculated as the slope of the linear regression of drug concentration in the dialysate $\left(\mathrm{C}_{\mathrm{d}}\right)$ as a function of drug concentration in the medium $\left(\mathrm{C}_{\mathrm{m}}\right.$; Equation 2$)$.

$$
\mathrm{RR}=\mathrm{C}_{\mathrm{d}} / \mathrm{C}_{\mathrm{m}} \times 100
$$

For the retrodialysis studies, the probe was perfused with STD solutions containing different concentrations of psoralen (Table 2). A linear probe was placed in a $50 \mathrm{~mL}$ beaker with the membrane portion completely immersed in ethanol solution $(10 \%, v / v)$ at room temperature. STD solutions were used as perfusates at a flow rate of $3.33 \mu \mathrm{L} /$ minutes. After equilibration for 30 minutes, the dialysate sample was collected into a vial for another 30 minutes. The diffusive loss of psoralen from perfusates was determined, and RR was calculated using Equation 3, where $\mathrm{C}_{\mathrm{p}}$ denotes the drug concentration in the perfusate and $\mathrm{C}_{\mathrm{d}}$ in the dialysate.

Table 2 Recovery of psoralen from a microdialysis probe (in vitro: $n=3$; in vivo: $n=5$ )

\begin{tabular}{|c|c|c|c|c|c|c|}
\hline$C_{m}\left(C_{p}\right)(\mu g / m L)$ & $\mathrm{C}_{\mathrm{d}}{ }^{\mathrm{a}}(\mu \mathrm{g} / \mathrm{mL})$ & $\mathbf{R R}^{\mathbf{a}}(\%)$ & $C_{p}-C_{d}{ }^{b}(\mu g / m L)$ & $\mathbf{R R}^{\mathrm{b}}(\%)$ & $C_{p}-C_{d}{ }^{c}(\mu g / m L)$ & $\mathbf{R R}^{\mathbf{c}}(\%)$ \\
\hline 2.016 & $0.841 \pm 0.012$ & $41.72 \pm 3.64$ & $0.945 \pm 0.113$ & $46.88 \pm 2.36$ & $0.901 \pm 0.024$ & $44.70 \pm 3.55$ \\
\hline 0.081 & $0.035 \pm 0.007$ & $43.83 \pm 1.95$ & $0.035 \pm 0.007$ & $44.00 \pm 1.58$ & $0.036 \pm 0.007$ & $45.15 \pm 2.16$ \\
\hline 0.202 & $0.087 \pm 0.014$ & $43.40 \pm 2.76$ & $0.092 \pm 0.010$ & $45.55 \pm 2.01$ & $0.088 \pm 0.009$ & $43.4 I \pm 2.74$ \\
\hline 0.403 & $0.173 \pm 0.020$ & $42.85 \pm 2.82$ & $0.201 \pm 0.032$ & $49.87 \pm 1.75$ & $0.172 \pm 0.031$ & $42.63 \pm 1.83$ \\
\hline 0.806 & $0.360 \pm 0.023$ & $44.63 \pm 1.49$ & $0.400 \pm 0.028$ & $49.59 \pm 1.34$ & $0.344 \pm 0.027$ & $42.66 \pm 2.06$ \\
\hline 0.040 & $0.017 \pm 0.001$ & $42.47 \pm 1.06$ & $0.018 \pm 0.004$ & $45.75 \pm 3.00$ & $0.020 \pm 0.003$ & $48.54 \pm 2.37$ \\
\hline
\end{tabular}

Notes: a Recovery of different psoralen standard solutions in vitro determined using $R R=C_{d} / C_{m} \times 100$. ${ }^{b}$ Recovery of different psoralen standard solutions with retrodialysisby-drug method in vitro determined by using Equation 3. 'Recovery of different psoralen standard solutions with retrodialysis-by-drug method in vivo determined by using Equation 3; RR ${ }^{a}$ compared with $R^{b}, P>0.05$.

Abbreviations: AUC, area under the concentration-time curve; $C_{d}$, drug concentration in the dialysate; $C_{m}$, drug concentration in the medium; $C_{\text {max }}$, peak concentration; $C_{p}$, drug concentration in the perfusate; ES, ethosomes; HPLC, high-performance liquid chromatography; min, minute; MRT, mean residence time; RR, relative recovery; $\mathrm{T}_{\text {max }}$, time to peak concentration. 


$$
\mathrm{RR}=\left(\mathrm{C}_{\mathrm{p}}-\mathrm{C}_{\mathrm{d}}\right) / \mathrm{C}_{\mathrm{p}}
$$

In vivo RR was determined using the retrodialysis-bydrug method, which relies on the assumption that net drug transport from the perfusate into the surrounding tissues through the microdialysis membrane is equal to that from the tissues into the perfusate. The diffusive loss of psoralen was determined, and RR was calculated using Equation 3. To determine the diffusive loss of psoralen, a rat was anesthetized with intraperitoneal urethane aqueous solution $(1.3 \mathrm{~g} / \mathrm{kg})$, and anesthesia was maintained throughout the experiment. The ambient temperature was kept constant at $25^{\circ} \mathrm{C}$. A linear probe was inserted into the dermis of the abdominal skin. After perfusion with ethanol solution $(10 \%$, $\mathrm{v} / \mathrm{v})$ for 1 hour, STD was used as the perfusate. HPLC assays were conducted to determine the loss of psoralen from the STD solutions.

\section{In vivo microdialysis studies}

Prior to administration of psoralen, the animals were anesthetized during in vivo microdialysis. The fur at the application site was shaved with a manual shaver in each rat. The following application sites were selected: the abdomen, chest, and scapulas, with the umbilicus, intermediate point of the two front paws, and shoulder blades as the centers, respectively. A microdialysis probe was implanted in the dermis, and the active dialysis window was placed immediately below the site of topical drug administration. The probe was continuously perfused with ethanol solution $(10 \%, \mathrm{v} / \mathrm{v})$. The skin was allowed to equilibrate for 1 hour before a blank sample was taken from the microdialysis probe, and 1.5 hours after the start of perfusion, either $1 \mathrm{~mL}$ of ethosomes or tincture was applied. A flat cylindrical plastic cover of about $1 \mathrm{~cm}$ in height, $1.5 \mathrm{~cm}$ in diameter, with an edge width of $2 \mathrm{~mm}$ was glued above the application site by using cyanoacrylate adhesive. During the experiment, the application site and the probe were kept level. Dialysate samples were collected into small vials, which were replaced every 30 minutes. Dialysis sampling was continued for 10 hours.

\section{Statistical analysis}

The results were expressed as mean \pm standard deviation. Statistical data were analyzed by one-way analysis of variance, and comparisons were made with the Tukey test using the SPSS software (version 13.0; IBM Corporation, Armonk, NY, USA). A $P$-value of less than $5 \%$ was considered significant. The cutaneous pharmacokinetic parameters of psoralen were calculated using WinNonLin (version 5.2; Pharsight
Corporation, Sunnyvale, CA, USA) and a noncompartmental analysis.

\section{Results and discussion Characterization of ethosomes}

The composition of ethosome formulations and the results of the experiments are presented in Table 1. The mean particle size of the ethosomes ranged from $56.71 \pm 1.02 \mathrm{~nm}$ to $159.07 \pm 25.97 \mathrm{~nm}$, as shown in Table 1. Ethosomes were relatively uniform in size because dynamic light scattering measurements indicated a narrow peak in the particle size distribution. According to the uniform design, a regression equation was drawn from the factors $\left(\mathrm{X}_{1}\right.$, Lipoid $\mathrm{S} 100[\mathrm{w} / \mathrm{v}, \%] ; \mathrm{X}_{2}$, ethanol [v/v, \%]) and response (Y, mean particle size $)$ as $\mathrm{Y}=-5.53 \mathrm{X}_{1}-3.14 \mathrm{X}_{2}+271.92(R$ [regression coefficient $]=0.83 ; \mathrm{S}$ [standard error] $=32.31, \mathrm{~F}=2.25$ ) using a multiple linear regression analysis. We did not observe any significant effects $\left(\{\mathrm{F}=2.25\}<\left\{\mathrm{F}_{0.05}[2,2]=19.00\right\}\right)$ of the different levels of Lipoid S 100 or ethanol on ethosome size. Excess PC and ethanol in the ethosome formulation may affect the distribution of particle size. ${ }^{26}$ In our study, the highest levels of Lipoid S $100(10 \%, \mathrm{w} / \mathrm{v})$ and ethanol (45\%, v/v) in ES5 were associated with the lowest particle size $(56.71 \pm 1.02 \mathrm{~nm})$. This is probably because a large amount of micelles were generated in ES5 with a small particle size distribution.

A representative micrograph of round ethosome vesicles from formulation ES2 is shown in Figure 1A. The vehicle structure is more evident in Figure 1B, showing two enlarged particles of psoralen ethosomes squeezed together. Because they were extruded together, the two ethosome particles were shaped like cocoons. Ethosomes with a small particle size and excellent deformability may easily penetrate the stratum corneum. ${ }^{26}$

\section{Ethosome formulation studies}

The uniform design was analyzed in vitro according to the EE $\left(\mathrm{Y}_{1}\right)$ and DSD $\left(\mathrm{Y}_{2}\right)$. Using a multiple linear

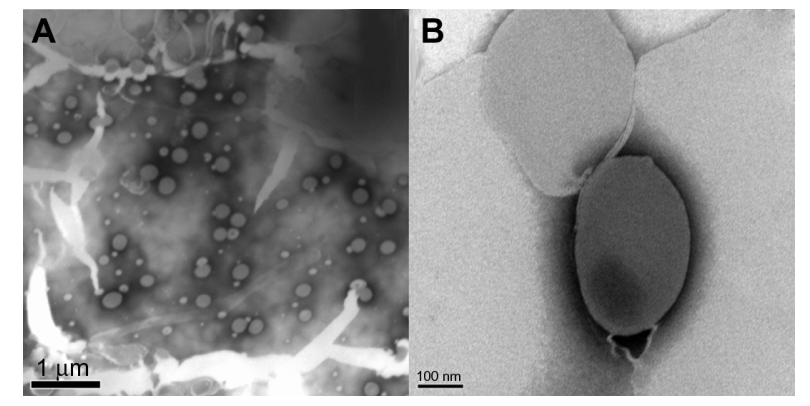

Figure I Transmission electron microscopy of psoralen-loaded ethosomes: (A) 15,000× magnification; (B) 100,000× magnification. 
regression analysis, the following regression equations were obtained:

$$
\begin{gathered}
\mathrm{Y}_{1}=-9.9 .98 \mathrm{X}_{1}-0.98 \mathrm{X}_{2}+145.06 \\
(R=0.78, \mathrm{~S}=27.61, \mathrm{~F}=1.54)
\end{gathered}
$$

and

$$
\begin{array}{r}
\mathrm{Y}_{2}=-0.10 \mathrm{X}_{1}+0.05 \mathrm{X}_{2}+0.83 \\
(R=0.23, \mathrm{~S}=1.90, \mathrm{~F}=0.05) .
\end{array}
$$

Both values of $\mathrm{F}$ were less than $\mathrm{F}_{0.05}(2,2)$, with the value 19.00 indicating that the regression equations were not statistically significant, and the arranged ethosome compositions had no significant effect on EE or DSD. An excessive use of ethanol or PC may prevent the formation of vesicles with the ability to encapsulate lipid-soluble molecules. ${ }^{27}$ In the present study, formulation ES5 with the highest Lipoid S 100 and ethanol contents and the lowest ethosome size showed the lowest EE $(18.30 \% \pm 2.29 \%)$ and an unsatisfactory level of DSD $\left(1.98 \pm 0.90 \mu \mathrm{g} / \mathrm{cm}^{2}\right)$. This was probably caused by the formation of a large number of micelles instead of ethosome vesicles, and hence, most of the drug molecules failed to be encapsulated in the vehicle. Since the aim of the current study was to improve the DSD of psoralen, an intuitive analysis of the uniform design resulted in the selection of ES2 formulation containing Lipoid $\mathrm{S} 100(5.0 \%, \mathrm{w} / \mathrm{v})$ and ethanol (40\%, v/v) for further studies. ES2 showed a satisfactory level of EE $(85.62 \% \pm 0.76 \%)$ and peak DSD of $3.61 \pm 1.21 \mu \mathrm{g} / \mathrm{cm}^{2}$ at 24 hours.

\section{In vitro skin permeation studies}

Compared to tincture, ethosomes enhanced the transdermal delivery of psoralen (Figure 2) and significantly increased the transdermal flux (Figure 3). The cumulative amount of psoralen penetrated across rat skin from the tested ethosome formulations was higher than from the compared tincture in the whole experimental period. The lag time of drug from the ethosomes through the skin was obviously shorter than that from the tincture, as can be seen in Figure 2. When ethanol is loaded in phospholipids, it can combine with the polar groups of the PC molecules and then lower the melting point of lipids in the stratum corneum, thus increasing lipid fluidity and cell membrane penetrability. Ethanol can also increase the flexibility of phospholipid vesicles and improve the ability of vesicles to deform, thereby allowing ethosomes to squeeze through skin channels that are smaller than the vehicle. ${ }^{28}$ Based on the generated data, the flexible lipid vesicles provided a superior enhancement of transdermal psoralen delivery to simply employing an ethanol solution as vehicle. The permeation rate of the optimal ethosome formulation (ES2) reached satisfactory levels $\left(38.89 \pm 3.22 \mu \mathrm{g} / \mathrm{cm}^{2} / \mathrm{h}\right)$ and showed no significant difference $(P>0.05)$ with that of $\operatorname{ES} 5\left(40.53 \pm 2.98 \mu \mathrm{g} / \mathrm{cm}^{2} / \mathrm{h}\right)$. Formulation ES1, with the lowest Lipoid S 100 and second-lowest ethanol levels, showed the lowest flux $\left(15.12 \pm 1.83 \mu \mathrm{g} / \mathrm{cm}^{2} / \mathrm{h}\right)$. The flux shown by ES1 was only slightly higher than that of the tincture $\left(13.51 \pm 0.88 \mu \mathrm{g} / \mathrm{cm}^{2} / \mathrm{h}\right)$, as shown in Figure 3 . The high ethanol concentration $(70 \%, \mathrm{v} / \mathrm{v})$ of the tincture was associated with enhanced skin permeability.

The ability of ethosomes to penetrate the skin is closely related to the proportion of excipients in the formulation. ${ }^{29}$ Ethosomes increase the mobility of skin lipids, allowing the drug to permeate deep into the skin and promoting cutaneous drug absorption. ${ }^{30}$ The DSD of psoralen from the

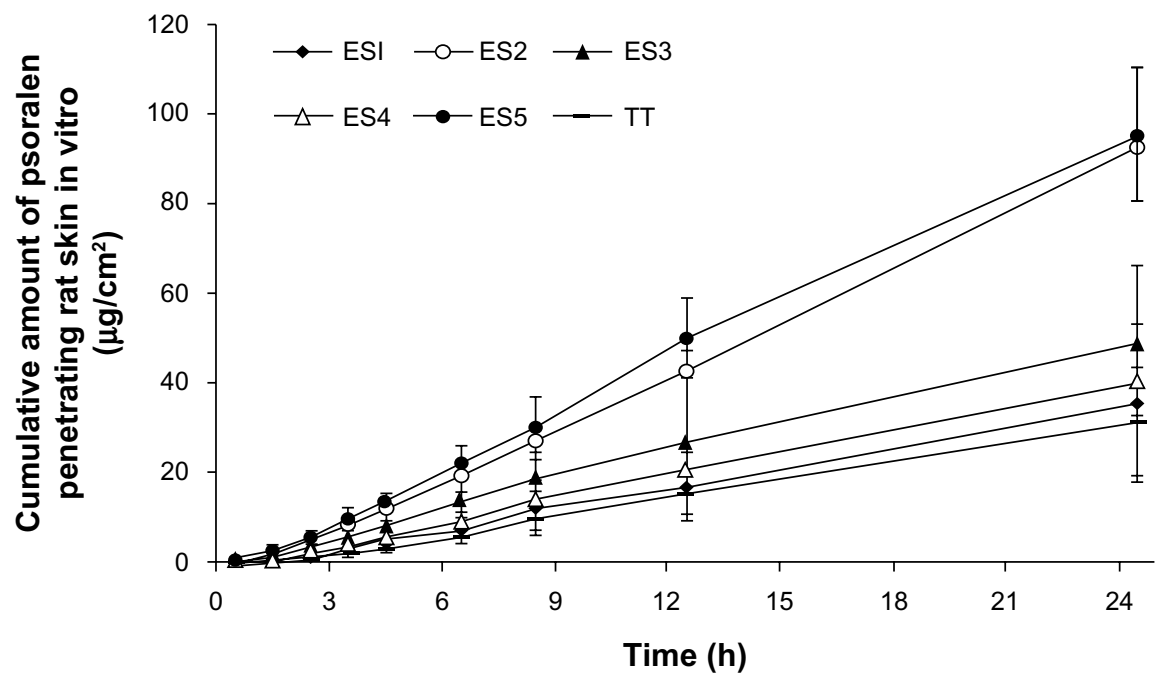

Figure 2 In vitro rat skin permeation profiles of psoralen from ethosomes and a tincture $(n=3)$.

Abbreviations: ES, ethosomes; h, hours; TT, tincture. 


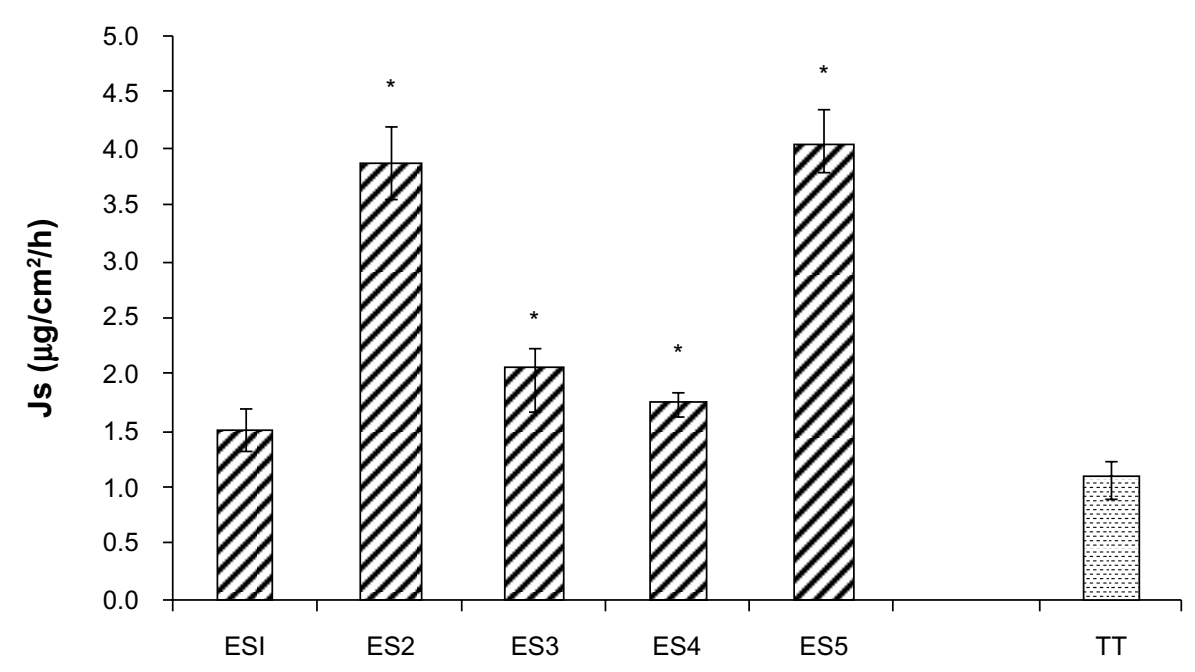

Figure 3 In vitro ratios of psoralen permeated through excised rat skin from ethosomes (ES2) and a tincture. Js: $* P<0.05$ versus tincture ( $n=3$ ). Abbreviations: ES, ethosomes; h, hours; Js, transdermal flux, TT, tincture.

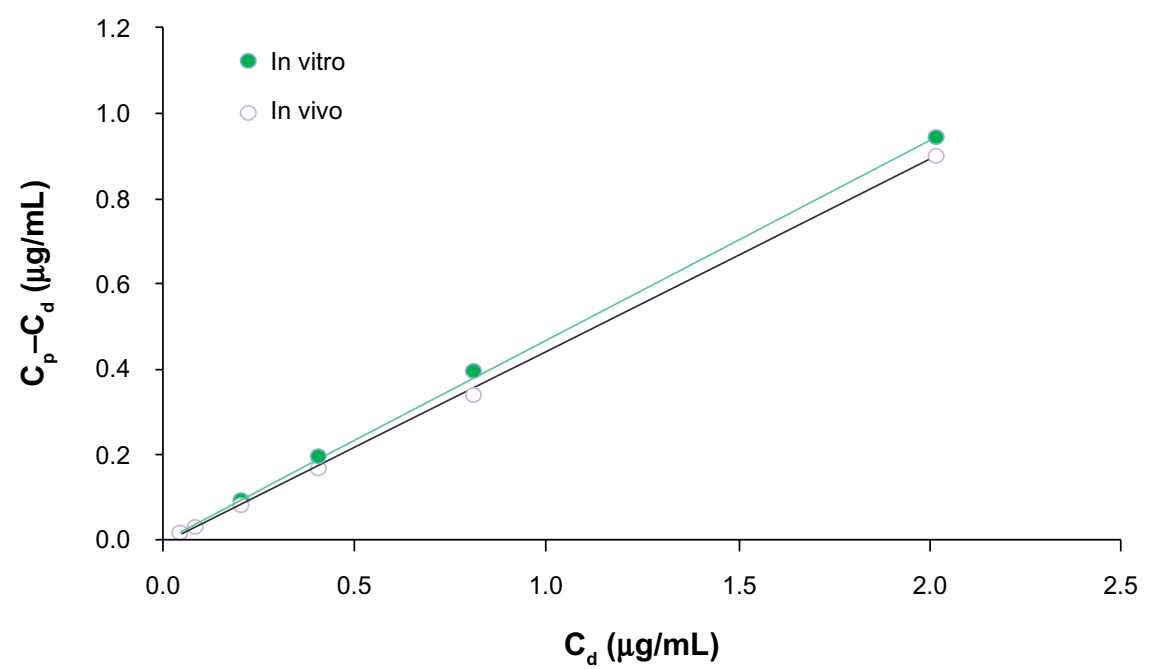

Figure $4 C_{p}$ as determined by the retrodialysis-by-drug method in vitro and in vivo.

Abbreviations: $C_{d}$, psoralen concentration in the dialysate; $C_{p}$, psoralen concentration in the perfusate.

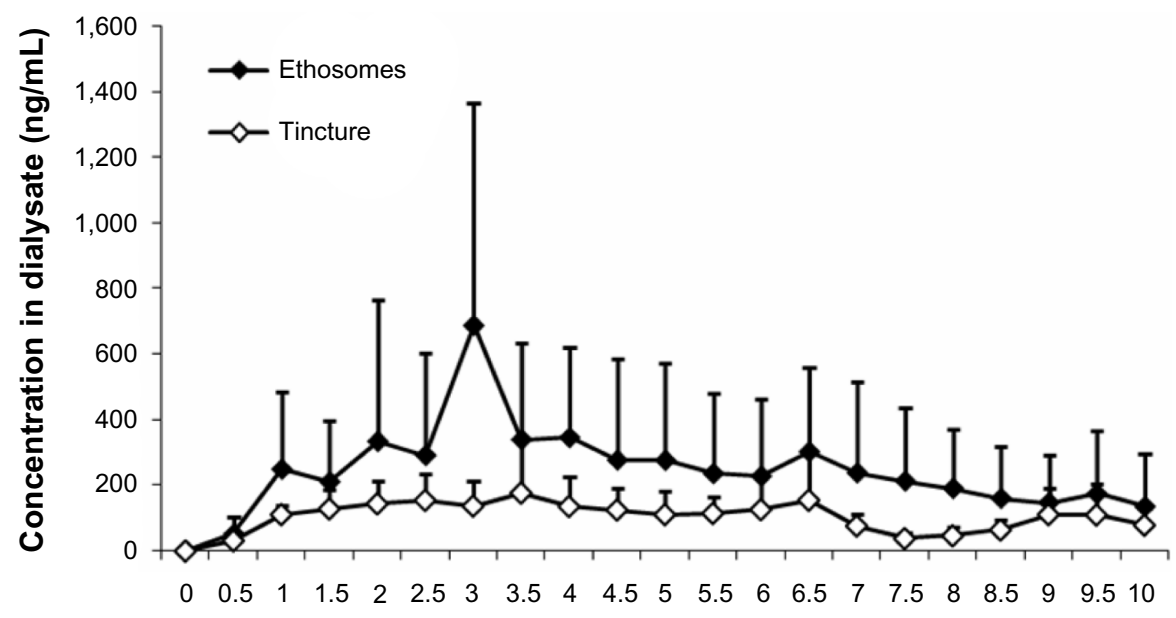

Time (hours)

Figure 5 Ten-hour time course of psoralen concentration after application of drug-containing ethosomes (ES2) and tincture to the abdominal skin of Sprague Dawley rats in vivo $(n=5)$. 
Table 3 Pharmacokinetic parameters of psoralen loaded in ethosomes (ES2) or tincture after application to rat abdomen skin in vivo $(n=5)$, as determined by microdialysis and HPLC

\begin{tabular}{llcc}
\hline Parameter & Unit & Ethosomes & Tincture \\
\hline $\mathrm{T}_{\max }$ & $\mathrm{min}$ & $180.00 \pm 0.00$ & $210.00 \pm 0.00$ \\
$\mathrm{C}_{\max }$ & $\mu \mathrm{g} / \mathrm{mL}$ & $688.76 \pm 25.33$ & $177.04 \pm 12.52^{*}$ \\
$\mathrm{AUC}_{0-\mathrm{t}}$ & $\mathrm{min} / \mu \mathrm{g} / \mathrm{mL}$ & $150,476.85 \pm 8,788.62$ & $64,180.5 \mathrm{I} \pm 1,909.86^{*}$ \\
$\mathrm{MRT}_{0-\mathrm{t}}$ & $\mathrm{min}$ & $283.00 \pm 15.48$ & $288.01 \pm 20.37$ \\
\hline
\end{tabular}

Note: Compared with the pharmacokinetic parameters from ethosomes, $* P<0.05$. Abbreviations: AUC, area under the concentration-time curve; $C_{\max }$, peak concentration; ES, ethosomes; HPLC, high-performance liquid chromatography; min, minute; $M R T$, mean residence time; $T_{\text {max }}$, time to peak concentration.

optimal ethosome formulation (ES2) was 6.56-fold higher $\left(3.61 \pm 1.21 \mu \mathrm{g} / \mathrm{cm}^{2}\right)$ than that from the tincture (Table 1). Of the ethosome formulations tested, only ES1 showed a slightly lower DSD than that of the tincture (Table 1). Currently, psoralen is the most common drug applied as a tincture in clinical practice. However, both permeability and DSD of the tincture were unsatisfactory after topical application. Therefore, the optimized ethosomes identified in our study may be used as a superior transdermal delivery carrier for psoralen.

\section{In vivo skin microdialysis}

Psoralen absorption after topical application of ethosomes and tincture was investigated in vivo by using skin microdialysis. Moreover, the concentration of psoralen was measured at different skin sites after topical application of ethosomes. There were no significant differences $(P>0.05)$ in in vitro RR between the dialysis and retrodialysis methods, indicating that the retrodialysis method was suitable for in vivo RR studies (Table 2). The in vitro and in vivo RR of the probe were maintained at the approximate level of $40 \%$ throughout the study. In the in vitro and in vivo RR validation studies, which used STD as perfusates, there was a linear correlation between drug concentration in the perfusate and drug loss from the perfusate (Figure 4: in vitro: $\left[\mathrm{C}_{\mathrm{p}}-\mathrm{C}_{\mathrm{d}}\right]=0.341 \mathrm{C}_{\mathrm{p}}-0.085$, $r^{2}=0.999$; in vivo: $\left.\left[\mathrm{C}_{\mathrm{p}}-\mathrm{C}_{\mathrm{d}}\right]=0.446 \mathrm{C}_{\mathrm{p}}+0.003, r^{2}=0.999\right)$. This correlation implies that in vitro and in vivo RR by retrodialysis are independent of the perfusate concentration, which indicates that it is possible to detect the true unbound extracellular level of psoralen by using the microdialysis technique.

Psoralen concentrations in the abdominal skin after topical application of ES2 or tincture are shown in Figure 5 and Table 3. During the initial 30 minutes, the drug concentration was not significantly different between the formulations. After 30 minutes, the higher drug concentration in the skin was obtained with the ethosome formulation, with the peak concentration $\left(\mathrm{C}_{\max }\right)$ observed 3 hours after application. The drug concentration at the site where ethosomes were applied first increased and then decreased. In contrast, the psoralen concentration after tincture application was relatively moderate from 2 hours to 6 hours after application, and the drug showed simple diffusion behavior during percutaneous absorption, which conformed to Fick's first law of diffusion. After tincture application, the drug in the dermal layer stabilized within a short period of time and remained relatively constant throughout the study. In contrast, drug molecules carried into the skin by ethosomes need to be released from the vehicles before being absorbed by the capillaries, which may result in fluctuations of the drug levels in the skin. During the study, compared

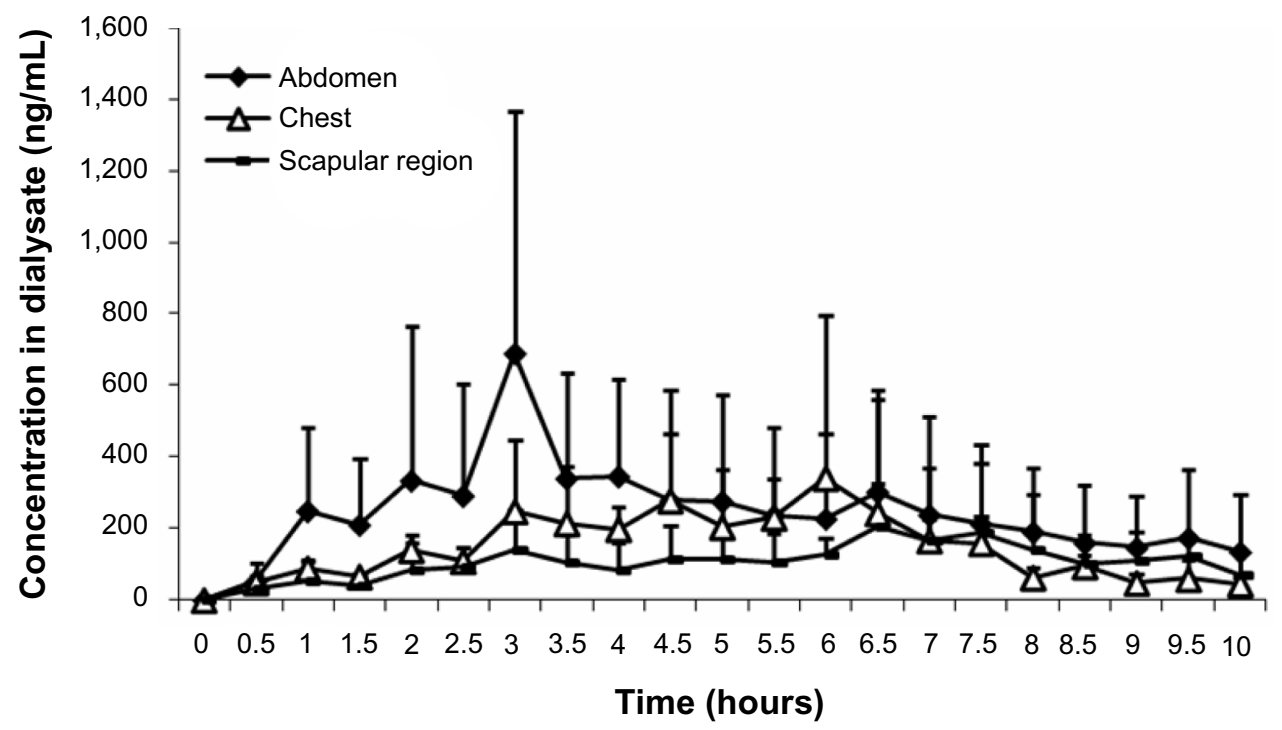

Figure 6 Ten-hour time course of psoralen concentration after ethosome application of drug-containing ethosomes (ES2) to the abdomen, scapularis, and chest regions of Sprague Dawley rats in vivo $(\mathrm{n}=5)$. 
Table 4 Pharmacokinetic parameters of psoralen loaded in ethosomes (ES2) after application to different regions of rat skin in vivo $(\mathrm{n}=5)$, as determined by microdialysis and HPLC

\begin{tabular}{llcrr}
\hline Parameter & Unit & Abdomen & Chest & Scapular region \\
\hline$T_{\max }$ & $\mathrm{min}$ & $180.00 \pm 17.25$ & $360.00 \pm 14.26^{*}$ & $390.00 \pm 16.83^{*}$ \\
$\mathrm{C}_{\max }$ & $\mu \mathrm{g} / \mathrm{mL}$ & $688.76 \pm 25.33$ & $339.61 \pm 21.03^{*}$ & $204.19 \pm 18.43^{*}$ \\
$\mathrm{AUC}_{0-\mathrm{t}}$ & $\mathrm{min} / \mu \mathrm{g} / \mathrm{mL}$ & $150,476.84 \pm 8,788.62$ & $90,295.68 \pm 1,145.29 *$ & $64,027.55 \pm 2,233.56 *$ \\
$\mathrm{MRT}_{0-\mathrm{t}}$ & $\mathrm{min}$ & $283.00 \pm 15.48$ & $299.64 \pm 15.36$ & $346.41 \pm 17.72 *$ \\
\hline
\end{tabular}

Note: Compared with the pharmacokinetic parameters from abdomen, $* P<0.05$.

Abbreviations: AUC, area under the concentration-time curve; $C_{\max }$, peak concentration; min, minute; ES, ethosomes; HPLC, high-performance liquid chromatography; MRT, mean residence time; $\mathrm{T}_{\max }$, time to peak concentration.

to tincture application, ethosome application was associated with a higher psoralen concentration, and the topical area under the concentration-time curve $\left(\mathrm{AUC}_{0-\mathrm{t}}\right)$ of the ethosomes was 2.34-fold higher than that of the tincture (Table 3).

After penetration through the epidermis, intercellular transport may be an important channel for the transit and absorption of ethosomes. ${ }^{31}$ Ethosomes may accumulate in the dermis after application to the skin surface. ${ }^{25,32}$ Microdialysis probes were precisely implanted in the dermis to accurately measure drug molecules in the extracellular fluid of the dermal layer where the ethosomes accumulated. Unlike the ethosomes, psoralen molecules from the tincture passively diffused through the stratum corneum and permeated into the epidermis. Most of the free drug molecules were captured by the capillary network under the epidermis and rapidly transferred into the microcirculation, while a few psoralen molecules hardly permeated into the deep skin and were detected by the microdialysis method.

As shown in Figure 5, psoralen concentrations in the skin after application of all formulations tended to be similar after 9 hours. However, in the in vitro study, skin deposition of the drug from ES2 continued to be notably higher than that of the tincture for 24 hours. For conventional vehicles, percutaneous drug absorption is hindered mainly by the stratum corneum. However, the ability of ethosomes to deform helps overcome this barrier and accumulate in the dermis. The dermal layer is the major component of the skin. It is composed of collagen, elastin, reticulin, and amorphous matrix and contains a large number of capillaries, including lymphatic capillaries, skin appendages, such as hair follicles and sebaceous glands, and even muscles. Because the dermal layer of excised rat skin is no longer functional, ethosomes permeation behavior in the dermal layer in vitro may differ from that in vivo, particularly in view of the fact that ethosomes permeate deep into the skin.

The changes in psoralen concentrations after drug application at different skin sites are presented in Figure 6. The drug concentration in the chest and scapular region increased slowly but showed a pattern similar to that observed on application to the abdomen. Psoralen applied to the abdomen reached $\mathrm{C}_{\max }$ at 3 hours, while that applied to the chest and the scapular region reached $\mathrm{C}_{\max }$ at 6 hours and at 6.5 hours, respectively, after application (Table 4). The $\mathrm{AUC}_{0-\mathrm{t}}$ in the abdomen was higher than those in the chest and scapular regions. The differences in permeability at these sites may result from the differences in skin structure, such as skin thickness or subcutaneous microvasculature. ${ }^{33,34}$

\section{Conclusion}

Ethosomes provide a novel approach for transdermal delivery of psoralen. They are characterized by much better percutaneous permeation than conventional vehicles. In vivo microdialysis is suitable for the evaluation of psoralen release from ethosomes into the deep skin, and the drug concentration gained from the ethosomes in dermis was obviously higher than that from the compared tincture during the experimental period. Enhanced permeation and skin deposition of psoralen delivered by ethosomes may help reduce toxicity and improve the efficacy of long-term psoralen treatment.

\section{Acknowledgments}

This work was financially supported by project (NCET080898) from the State Education Ministry of the People's Republic of China, National Natural Science Fund of China (81303234), and Youth Scientific Research Fund (20134q098) from Shanghai Health Bureau.

\section{Disclosure}

The authors report no conflicts of interest in this work.

\section{References}

1. Ada S, Güleç AT, Tülin Güleç A. CD8+ poikilodermatous mycosis fungoides with a nonaggressive clinical behaviour and a good response to psoralen plus ultraviolet A treatment. Br J Dermatol. 2007;157(5): 1064-1066.

2. Sapam R, Agrawal S, Dhali TK. Systemic PUVA vs narrowband UVB in the treatment of vitiligo: a randomized controlled study. Int $J$ Dermatol. 2012;51(9):1107-1115. 
3. Stern RS. Psoralen and ultraviolet a light therapy for psoriasis. $N$ Engl J Med. 2007;357(7):682-690.

4. Almutawa F, Alnomair N, Wang Y, Hamzavi I, Lim HW. Systematic review of UV-based therapy for psoriasis. Am J Clin Dermatol. 2013;14(2):87-109.

5. Bacigalupi RM, Postolova A, Davis RS. Evidence-based, non-surgical treatments for vitiligo: a review. Am J Clin Dermatol. 2012;13(4): 217-237.

6. Umar N, Yamamoto S, Loerbroks A, Terris D. Elicitation and use of patients' preferences in the treatment of psoriasis: a systematic review. Acta Derm Venereol. 2012;92(4):341-346.

7. Ozkan I, Köse O, Ozmen I, Arca E. Efficacy and safety of non-laser, targeted UVB phototherapy alone and in combination with psoralen gel or calcipotriol ointment in the treatment of localized, chronic, plaquetype psoriasis. Int J Dermatol. 2012;51(5):609-613.

8. Lapolla W, Yentzer BA, Bagel J, Halvorson CR, Feldman SR. A review of phototherapy protocols for psoriasis treatment. JAm Acad Dermatol. 2011;64(5):936-949.

9. Nagase K, Hirashima N, Koba S, Inoue T, Misago N, Narisawa Y. Acquired dermal melanocytosis induced by psoralen plus ultraviolet $\mathrm{A}$ therapy. Acta Derm Venereol. 2012;92(6):691-692.

10. Stern RS; PUVA Follow-Up Study. The risk of squamous cell and basal cell cancer associated with psoralen and ultraviolet A therapy: a 30-year prospective study. J Am Acad Dermatol. 2012;66(4):553-562.

11. Choi MJ, Maibach HI. Elastic vesicles as topical/transdermal drug delivery systems. Int J Cosmet Sci. 2005;27(4):211-221.

12. Pierre MB, Dos Santos Miranda Costa I. Liposomal systems as drug delivery vehicles for dermal and transdermal applications. Arch Dermatol Res. 2011;303(9):607-621.

13. Touitou E, Dayan N, Bergelson L, Godin B, Eliaz M. Ethosomes novel vesicular carriers for enhanced delivery: characterization and skin penetration properties. J Control Release. 2000;65(3):403-418.

14. Ainbinder D, Paolino D, Fresta M, Touitou E. Drug delivery applications with ethosomes. J Biomed Nanotechnol. 2010;6(5):558-568.

15. Nanda A, Nanda S, Ghilzai NM. Current developments using emerging transdermal technologies in physical enhancement methods. Curr Drug Deliv. 2006;3(3):233-242.

16. Ajazuddin, Saraf S. Applications of novel drug delivery system for herbal formulations. Fitoterapia. 2010;81(7):680-689.

17. Chen JG, Liu YF, Gao TW. Preparation and anti-inflammatory activity of triptolide ethosomes in an erythema model. J Liposome Res. 2010;20(4): 297-303.

18. Gupta U, Jain NK. Non-polymeric nano-carriers in HIV/AIDS drug delivery and targeting. Adv Drug Deliv Rev. 2010;62(4-5):478-490.

19. Zhang Z, Wo Y, Zhang Y, et al. In vitro study of ethosome penetration in human skin and hypertrophic scar tissue. Nanomedicine. 2012;8(6): $1026-1033$.

20. Zhang X, Liu L, Zhang X, et al. Analytical methods for brain targeted delivery system in vivo: perspectives on imaging modalities and microdialysis. J Pharm Biomed Anal. 2012;59:1-12.
21. Cheng GW, Hsu KC, Lee CF, Wu HL, Huang YL. On-line microdialysis coupled with liquid chromatography for biomedical analysis. J Chromatogr Sci. 2009;47(8):624-630.

22. Holmgaard R, Nielsen JB, Benfeldt E. Microdialysis sampling for investigations of bioavailability and bioequivalence of topically administered drugs: current state and future perspectives. Skin Pharmacol Physiol. 2010;23(5):225-243.

23. Kreilgaard M. Dermal pharmacokinetics of microemulsion formulations determined by in vivo microdialysis. Pharm Res. 2001;18(3): 367-373.

24. Maestrelli F, Capasso G, González-Rodríguez ML, Rabasco AM, Ghelardini C, Mura P. Effect of preparation technique on the properties and in vivo efficacy of benzocaine-loaded ethosomes. J Liposome Res. 2009;19(4):253-260.

25. Dubey V, Mishra D, Jain NK. Melatonin loaded ethanolic liposomes: physicochemical characterization and enhanced transdermal delivery. Eur J Pharm Biopharm. 2007;67(2):398-405.

26. Paolino D, Lucania G, Mardente D, Alhaique F, Fresta M. Ethosomes for skin delivery of ammonium glycyrrhizinate: in vitro percutaneous permeation through human skin and in vivo anti-inflammatory activity on human volunteers. J Control Release. 2005;106(1-2):99-110.

27. Fang YP, Tsai YH, Wu PC, Huang YB. Comparison of 5-aminolevulinic acid-encapsulated liposome versus ethosome for skin delivery for photodynamic therapy. Int J Pharm. 2008;356(1-2):144-152.

28. Verma P, Pathak K. Therapeutic and cosmeceutical potential of ethosomes: An overview. J Adv Pharm Technol Res. 2010;1(3): 274-282.

29. Celia C, Cilurzo F, Trapasso E, Cosco D, Fresta M, Paolino D. Ethosomes ${ }^{\circledR}$ and transfersomes ${ }^{\circledR}$ containing linoleic acid: physicochemical and technological features of topical drug delivery carriers for the potential treatment of melasma disorders. Biomed Microdevices. 2012;14(1):119-130.

30. Dubey V, Mishra D, Dutta T, Nahar M, Saraf DK, Jain NK. Dermal and transdermal delivery of an anti-psoriatic agent via ethanolic liposomes. $J$ Control Release. 2007;123(2):148-154.

31. Jain S, Tiwary AK, Sapra B, Jain NK. Formulation and evaluation of ethosomes for transdermal delivery of lamivudine. AAPS Pharm Sci Tech. 2007;8(4):E111.

32. Rao Y, Zheng F, Zhang X, Gao J, Liang W. In vitro percutaneous permeation and skin accumulation of finasteride using vesicular ethosomal carriers. AAPS Pharm Sci Tech. 2008;9(3):860-865.

33. Mills PC, Cross SE. Regional differences in the in vitro penetration of hydrocortisone through equine skin. JVet Pharmacol Ther. 2006;29(1): 25-30.

34. Mills PC, Magnusson BM, Cross SE. Investigation of in vitro transdermal absorption of fentanyl from patches placed on skin samples obtained from various anatomic regions of dogs. Am J Vet Res. 2004;65(12): $1697-1700$.
International Journal of Nanomedicine

\section{Publish your work in this journal}

The International Journal of Nanomedicine is an international, peerreviewed journal focusing on the application of nanotechnology in diagnostics, therapeutics, and drug delivery systems throughout the biomedical field. This journal is indexed on PubMed Central, MedLine, CAS, SciSearch ${ }^{\circledR}$, Current Contents ${ }^{\circledR} /$ Clinical Medicine,
Dovepress

Journal Citation Reports/Science Edition, EMBase, Scopus and the Elsevier Bibliographic databases. The manuscript management system is completely online and includes a very quick and fair peer-review system, which is all easy to use. Visit http://www.dovepress.com/ testimonials.php to read real quotes from published authors. 